\title{
Overriding stimulus-driven attentional capture
}

\author{
WILLIAM F. BACON and HOWARD E. EGETH \\ Johns Hopkins University, Baltimore, Maryland
}

\begin{abstract}
Theeuwes (1992) found a distracting effect of irrelevant-dimension singletons in a task involving search for a known target. He argued from this that selectivity is determined solely by stimulus salience; the parallel stage of visual processing cannot provide top-down guidance to the attentive stage sufficient to permit completely selective use of task-relevant information. We argue that in the task used by Theeuwes, subjects may have adopted the strategy of searching for an odd form even though the specific target form was known. In Experiment 1, we replicated Theeuwes's findings. Search for a circle target among diamond nontargets was disrupted by the presence of a diamond nontarget that was uniquely colored. In two subsequent experiments, we discouraged the singleton detection strategy, forcing subjects to search for the target feature. There was no distracting effect of a color singleton in these experiments, even with displays physically identical to those of Experiment 1, demonstrating that top-down selectivity is indeed possible during visual search. We conclude that goal-directed selection of a specific known featural identity may override stimulus-driven capture by salient featural singletons.
\end{abstract}

Considerable debate has erupted recently over the sources of control over the locus of visual attention (e.g., Folk, Remington, \& Johnston, 1992, 1993; Theeuwes, 1991a, 1992; Yantis, 1993a, 1993b). Traditionally, two sources of control have been recognized. Broadbent (1958) wrote that attentional selection is influenced both "by certain properties of the events and by certain states of the organism" (p. 297). In modern parlance these correspond to stimulus-driven and goal-directed selection, respectively (in the terminology of Yantis, 1993a). Stimulus-driven (or bottom-up) selection is said to occur when attention is captured by properties of the stimulus even if they are irrelevant to the current task. Goal-directed (or top-down) selection, on the other hand, is said to occur when the observer's knowledge or beliefs about the task determine what is selected in the visual field.

Under most circumstances, the distribution of attention across the visual field presumably reflects a combination of stimulus-driven and goal-directed processes. However, tasks can be contrived that apparently reveal just one mode of processing. For example, Yantis and Jonides (1984) found evidence for stimulus-driven selection based on abrupt onset. Subjects searched for a target letter among nontarget letters, a task that normally requires serial attentional scrutiny. On each trial, one letter had an abrupt

This research was supported by Grant DBS 89-19554 from the National Science Foundation and Grant AFOSR-ISSA-92-0041 from the Air Force Office of Scientific Research. We thank Brad Gibson, Anne Hillstrom, and Steven Yantis for helpful discussions concerning this research, and James Johnston, Jan Theeuwes, and Steven Todd for thoughtful comments on an earlier version of this paper. William Bacon is now at NASA Ames Research Center, Mail Stop 262-2, Moffett Field, CA 94035 (e-mail: bacon@eos.arc.nasa.gov). Howard Egeth is with the Department of Psychology, Johns Hopkins University, Baltimore, MD 21218 (e-mail: egeth@jhuvm.hcf.jhu.edu). onset. When the abrupt-onset letter was the target, search time was independent of the number of distractors, suggesting that the abrupt onset captured attention in a stimulus-driven manner. Goal-directed processes were not implicated in the selection of the onset element because the onset was orthogonal to the observer's task (the onset element was no more likely than a no-onset element to be the target letter). ${ }^{1}$

A demonstration of a purely goal-directed allocation of attention is provided by Posner, Snyder, and Davidson (1980). Using symbolic cues (digits presented at fixation, designating a particular display location likely to contain the subsequent target), Posner et al. found that subjects could covertly direct attention to a circumscribed region of the visual field. This ability revealed itself in benefits (faster target detection times) when the cue was valid, and in costs (slower target detection times) when the cue was invalid. This variety of attentional allocation (goal-directed attention to a spatial location) is relatively uncontroversial. What is disputed is whether attention can be directed toward objects not in a particular spatial location, but possessing certain features, such as a particular color or shape (Cave \& Wolfe, 1990; LaBerge \& Brown, 1989; Pashler, 1988; Theeuwes, 1991a, 1992; Treisman, 1988; Wolfe, Cave, \& Franzel, 1989). This is one of the central questions of the present study.

A promising approach for understanding goal-directed and stimulus-driven attentional processes is to examine performance in tasks that pit the two processes against each other. For example, when a task involves both a valid symbolic cue and an uninformative abrupt onset, the goaldirected allocation of spatial attention induced by the symbolic cue overrides the attentional capture by the abrupt onset. In other words, observers are not distracted by an abrupt onset when they are in a spatially focused attentional state (Theeuwes, 1991b; Yantis \& Jonides, 1990). 
Extending this logic, Theeuwes (1991a) examined whether goal-directed attention toward a particular stimulus dimension could override stimulus-driven distraction caused by a singleton in an irrelevant dimension. In the Theeuwes (1991a) experiments, subjects were in a spatially diffuse attentional state, in that they were responsible for many display locations; under investigation was whether attention could be directed to elements according to task-relevant stimulus dimensions such as color and shape. In one experiment, five, seven, or nine colored shapes (circles or diamonds) were presented around the circumference of an imaginary circle centered on fixation. (See Figure 1 for a depiction of similar displays from the present experiments; the caption describes how these displays differ from those used by Theeuwes.) Centered inside each shape was a line segment, either horizontal or vertical (in the target shape) or oblique (in the distractor shapes). In one condition, the target shape was defined by its unique form. Thus, the target was unpredictably either a circle among diamond distractors or a diamond among circle distractors. Subjects discriminated the orientation of the line (horizontal vs. vertical) inside the target shape. Theeuwes found that when one of the distractor shapes was a color singleton (e.g., when the target was a green circle, one of the green distractor diamonds was replaced by a red diamond), response times were elevated. Apparently, a singleton in a dimension known to be irrelevant can capture attention. In addition, Theeuwes (1991a, Experiment 3) found that when the form difference (circle vs. square) was more salient than the color difference (yellowish red vs. yellowish green), a distraction effect was evident when the roles of the dimensions were reversed. That is, subjects searching for the element with the unique color were distracted by the presence of a single distractor element with a unique form. On the basis of these results, Theeuwes argued that topdown (goal-directed) selection of a particular stimulus dimension was not possible: Elements were selected for processing solely on the basis of their bottom-up difference signals (i.e., their salience). When the color difference was more salient than the form difference, the uniquely colored element was selected first, even when color was known to be irrelevant.

The findings of Theeuwes (1991a) suggest a relative impotence of goal-directed attentional selection. However, in those experiments, goal-directed selection could only be based on the particular stimulus dimension known to be relevant. This leaves open the possibility that top-down control may be more effective in overriding bottom-up processes when attention can be directed to a particular stimulus value. In order to examine this possibility, Theeuwes (1992) investigated whether top-down selection of a particular stimulus value could override bottomup interference from irrelevant distractor singletons. In these experiments, subjects knew not only the dimension (e.g., form) that was relevant for selection, but also the particular value on that dimension that defined the target. Specifically, subjects always searched for a green circle among known distractor shapes. In the "form'" condition, the distractors were green diamonds (so that the target had a known unique shape). In the "color" condition, the distractors were red circles (so that the target had a known unique color). As in Theeuwes (1991a), subjects discriminated the orientation of a line inside the target shape; of interest was the distracting effect of a singleton in the irrelevant dimension (e.g., in the "form" condition, one of the green diamonds appearing in red). In these experiments, subjects presumably could search for a specific colored shape, a green circle, rather than for a unique color or shape. As in Theeuwes's earlier experiments, the presence of a salient singleton in the irrelevant dimension interfered with performance, elevating response times (RTs) in this case by about $15-25 \mathrm{msec}$. Theeuwes (1992) argued that the finding of a distraction effect under these circumstances suggests that top-down selectivity for color or shape is not possible: Even when the specific target features are known, selection is determined solely by bottom-up (stimulus-driven) factors. That is, items are selected in order of their salience.

Other findings in the literature make the claims of Theeuwes (1991a, 1992) surprising. Similarly to Theeuwes (1991a), Pashler (1988, Experiment 6) had found that an irrelevant color singleton interfered substantially with a form singleton detection task when the target form was unspecified. However, when Pashler's subjects searched for a known form target, there was little or no effect of an irrelevant color singleton. ${ }^{2}$ Pashler accounted for his findings (as well as for apparent inconsistencies in the texture segregation literature) in the following way. He suggested that a known target can be detected in one of two ways. First, an observer may monitor a specific feature map (as in Treisman \& Souther, 1985) that codes for the presence of the relevant feature. Alternatively, an observer may rely on a mode of processing that identifies elements that differ from their backgrounds. We refer to these two modes of processing as the feature search mode and the singleton detection mode, respectively (LaBerge \& Brown, 1989, make a similar distinction; see also Bravo \& Nakayama, 1992). Note that when the target feature is known, both of these strategies are available. However, when the target feature is unknown, only the latter strategy is available. Thus, in order to account for Pashler's results, one need only assume that the feature search mode is not susceptible to interference from variation in an irrelevant dimension, but that the singleton detection mode is susceptible to such interference. This assumption seems reasonable. Using the feature search strategy, observers presumably monitor a retinotopic feature map, and direct attention to the location of the element that generates activation in that map. There is no reason to suspect that the coding of features in maps corresponding to one dimension would be influenced by variation in some other, irrelevant dimension. On the other hand, the singleton detection strategy relies on detecting elements that differ from their surrounds. It may be that the process that computes feature differences cannot be restricted to a given 
dimension, but simply indicates the overall salience of each element; only after a highly salient element has been selected is it possible to determine the dimension on which the element is unusual. This view of the nature of preattentive processing had an early voice in Julesz (1981). It forms the basis for the "bottom-up" component of the guided search model (Cave \& Wolfe, 1990) and is consistent with the work of Koch and Ullman (1985), Nothdurft (1992), and Sagi and Julesz (1984).

It is worth considering how these two proposed search modes relate to recent theoretical positions concerning bottom-up versus top-down control of visual attention. Theeuwes (1991a, 1992) resides at one end of the theoretical spectrum, arguing that the locus of attention is controlled solely by bottom-up factors. This position is equivalent to arguing that only the second of Pashler's (1988) two modes of processing-singleton detectionis actually available to an observer. The feature search mode of processing relies on top-down information about the specific featural identity of the target, and so by Theeuwes's account would not be possible.

At the other end of the theoretical spectrum is the "contingent involuntary orienting hypothesis" of Folk et al. (1992). According to Folk et al., whether or not a salient task-irrelevant stimulus will capture attention depends on the observer's attentional control settings. This position stands in stark contrast to Theeuwes's (1991a, 1992) purebottom-up hypothesis, because under the contingent involuntary orienting hypothesis attentional allocation is continuously influenced by top-down factors. In support of their hypothesis, Folk et al. found that a spatially incompatible precue interfered with subsequent target identification only when the precue and target were both defined by the same type of discontinuity. For example, when the precue display consisted of white markers at three stimulus locations and a red marker at the fourth location, the precue was defined as a static (color) discontinuity. A spatially incompatible precue of this type interfered with identification of a subsequent target only when the target was itself defined as a static (color) discontinuity, and not when the target was defined by a dynamic discontinuity, such as abrupt onset. From this and other experiments, Folk et al. argue that involuntary shifts of attention are never completely bottom-up, because they are modulated by the organism's attentional state, which is a function of the current behavioral goals.

Folk et al.'s (1992) position contains a natural explanation for the assertion described above that the singleton detection mode is vulnerable to interference from irrelevant singletons, but that the feature search mode is not. In the framework of the contingent involuntary orienting hypothesis, being in singleton detection mode would correspond to having an attentional state that is so broad that the irrelevant distractor fulfills the criterion defining the target item (i.e., "find the discrepant item"). The feature search mode would correspond to having a narrower atten- tional control setting, one that included the target but not the irrelevant distractor.

If indeed there are two search modes, one that depends on the specific featural property (and is invulnerable to irrelevant singletons) and another that identifies salient elements without regard for identity or even dimension, Theeuwes's (1992) results are puzzling. Because the target identity was known, it is natural to expect that observers would have employed the feature search mode and would not have been distracted by irrelevant singletons. The finding that irrelevant singletons did interfere suggests several possibilities. First, Theeuwes's (1991a, 1992) conclusion may be correct: Perhaps parallel search is always mediated by bottom-up difference signals, and top-down selectivity for a specific color or shape is not possible. This would correspond to the claim that the feature search mode as described above is not a viable means of directing visual attention. Another possibility is that even in the feature search mode, stimulus selection is determined by a combination of top-down and bottom-up factors (cf. Cave \& Wolfe, 1990). That is, perhaps elements coded on the known-to-be-relevant feature map receive extra activation on an attentional priority map, but bottom-up differences also contribute to the attentional priority map. Under some circumstances (such as those in Theeuwes's experiments) these bottom-up difference signals may be so large as to mask the top-down identity-specific activations. A final possibility is that subjects treated the task as a singleton detection task, even though the feature search strategy was available. For example, subjects in the "form" condition (circle target among diamond distractors) may have searched not for the circle, but for the odd form. Under this scenario, complete top-down selectivity may indeed be possible, but it was not found in the Theeuwes (1992) experiments because, for whatever reason, subjects used the singleton detection strategy, which is susceptible to interference from irrelevant singletons. This final possibility was tested in the present experiments. Note that if this dual-strategy hypothesis is supported, it would lead us to reject the first possibility (Theeuwes's pure-bottomup hypothesis) described above: That is, if we find that under the right circumstances subjects are able to perform a parallel search in a way that is immune to distraction from irrelevant singletons, we would reject the claim that top-down selectivity of a specific stimulus value is not possible. On the other hand, the dual-strategy hypothesis and the other hypothesis discussed above (that bottomup signals may overwhelm top-down activation) are not mutually exclusive, and so evidence in support of the dualstrategy hypothesis would not disconfirm the hypothesis of overwhelmed top-down processing.

In order to test the hypothesis that the interference effects found in Theeuwes (1992) are due to subjects' adopting a singleton detection strategy, we modified Theeuwes's task so as to make such a strategy unattractive. First, however, it was necessary to replicate the basic effect of inter- 
ference from an irrelevant singleton during parallel search for a known target.

\section{EXPERIMENT 1}

In our first experiment, we attempted to replicate the basic effect Theeuwes (1992) found in the "form condition" of his Experiment 1A. Subjects in Theeuwes's form condition searched for a green circle among green diamonds, and were apparently distracted by the presence of an irrelevant color singleton. ${ }^{3}$ We made only one substantive change in the experimental procedure. In Theeuwes's experiments, the line inside the target shape was either horizontal or vertical, but the lines inside the nontarget shapes were tilted $22.5^{\circ}$ to either side of horizontal or vertical. Under these circumstances, the defining and reporting attributes (Duncan, 1985) were not necessarily different, because subjects could conceivably respond as soon as they detected a horizontal or vertical line, regardless of the shape it was contained in. Thus, in our experiment the nontarget lines were (randomly) horizontal or vertical, so that a correct response depended on actually identifying the target shape. Otherwise, our experimental conditions were as similar as possible to those of Theeuwes.

\section{Method}

Subjects. Sixteen Johns Hopkins University undergraduate and graduate students ( 8 male) were recruited for the 50-min experiment. Each subject reported normal or corrected-to-normal visual acuity and normal color vision. The subjects were paid $\$ 5$ for their participation.

Apparatus. Displays were generated by a Zeos 486 SLC computer on a Zeos VGA color monitor, using $640 \times 480$ resolution graphics mode. Responses were collected via the computer keyboard.

Stimuli. Stimulus displays consisted of five, seven, or nine colored shapes presented equally spaced along, and centered on, the circumference of an imaginary circle (3-cm radius) centered at fixation. Thus, display density varied with number of shapes. At a typical viewing distance of $50 \mathrm{~cm}$, the centers of the shapes were $3.4^{\circ}$ from fixation. The target shape was a circle $1.4^{\circ}$ in diameter, presented in green (CIE coordinates .290/.599). Nontarget shapes were diamonds $\left(45^{\circ}\right.$ rotated squares) $1.6^{\circ}$ on a side, presented in the same color. In the distractor condition, one of the nontarget diamonds was presented in red (CIE coordinates .640/.330). These colors were selected so as to be equiluminant by the flicker criterion (Ives, 1912). Centered inside each shape was a white horizontal or vertical line segment $\left(0.5^{\circ}\right.$ in length). Fixation was marked with a small (each segment $0.2^{\circ}$ ) gray plus sign. Sample displays (with display size 7) are shown in Figure 1, for both the no-distractor and the distractor conditions.

Procedure. The subjects were instructed to determine the orientation (horizontal or vertical) of the line segment inside the green circle, and to respond by pressing designated keys on the computer keyboard as quickly as possible, while maintaining very high accuracy. Right-handed subjects pressed the " $Z$ " key for vertical and the "/" key for horizontal; this response mapping was reversed for left-handed subjects, so that all subjects responded to horizontal lines with their dominant hands. The subjects were informed of the shapes and colors of the target and nontarget elements, both verbally and through sample displays showing a typical trial in the no-distractor and distractor conditions. Eye movements were not monitored, but the subjects were encouraged to maintain fixation throughout the trial and reported no difficulty doing so. Trial presentation was force paced. Each trial began with the presentation

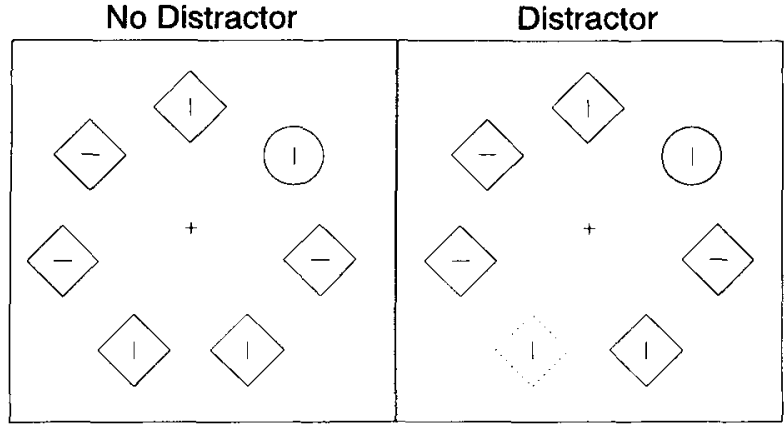

Figure 1. Sample stimulus displays (with display size 7) for Experiment 1. Left panel shows the no-distractor condition, in which the green circle target appears among green diamond nontargets. Right panel shows the distractor condition, in which one of the diamond nontargets is red. (Solid outlines were green; dotted outline was red; embedded horizontal and vertical line segments were white; background was black. Displays in Theeuwes's [1991a, 1992] form condition were very similar, except that the line segments in nontarget shapes were tilted $22.5^{\circ}$ from horizontal or vertical.)

of a fixation cross for $350 \mathrm{msec}$. This was followed by the stimulus display, which remained visible for $2,500 \mathrm{msec}$ or until response. The subjects responded by pressing one of the two keys on which their index fingers were resting. The computer recorded the RT to the nearest millisecond, and it beeped on trials in which a response error was made. Error trials were followed by an unanalyzed recovery trial (a repetition of a randomly selected trial from the current block) and were repeated later in the block. Consecutive trials were separated by a blank intertrial interval of $1,500 \mathrm{msec}$.

The main part of the experiment consisted of four blocks of 144 trials, the blocks alternating between the no-distractor and distractor conditions. Rests were allowed at the halfway point within each block, and at the end of each block. Half the subjects began with a no-distractor block; the other half began with a distractor block. The subjects first received a practice half block ( 72 unanalyzed trials) in each of the two conditions. In each block, there were equal numbers of trials at each of the three levels of display size $(5,7,9)$, and equal numbers of horizontal and vertical targets.

\section{Results and Discussion}

RTs greater than 2,000 msec were excluded from analysis; no subject lost more than $1 \%$ of trials as a result of this procedure. Mean correct RTs were entered into an analysis of variance (ANOVA) with distraction (no distractor vs. distractor) and display size $(5,7,9)$ as withinsubjects factors. There were main effects of both factors $[F(1,15)=24.42, p<.001$, for the distraction effect; $F(2,30)=7.59, p<.01$, for the display-size effect]. The interaction was not significant $[F(2,30)=2.29, p>.05]$. These results are depicted in Figure 2 . As is evident in the figure, the main effect of display size probably does not indicate serial search. To explore this, slopes were calculated for each subject. The mean slopes were $-1 \mathrm{msec} /$ element for the no-distractor condition and $3 \mathrm{msec} / \mathrm{element}$ for the distractor condition. The latter slope was significantly different from zero $[t(15)=1.81, p<.05$, onetailed]. It would be difficult to argue, however, that such a small slope reflects serial processing. 


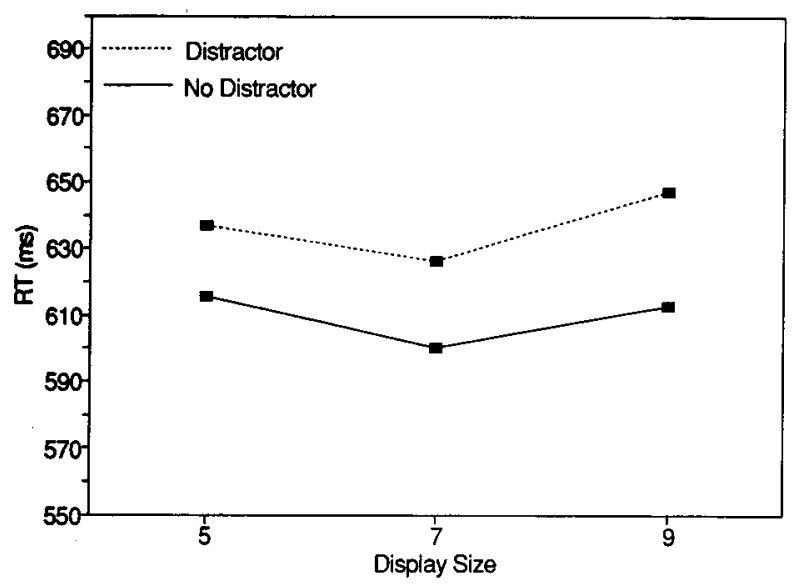

Figure 2. Mean response times, as a function of display size and distractor condition, for Experiment 1.

Error rates are shown in Table 1. The subjects' overall error rates ranged from $0.7 \%$ to $6.0 \%$, with a mean of $2.7 \%$. Error rates were submitted to an arcsine transformation (Winer, 1971) and entered into a $2 \times 3$ (distraction $\times$ display size) repeated measures ANOVA. There were no significant effects.

The results of this experiment are clear-cut. Using a task very similar to the form condition of Theeuwes (1992), we replicated his finding of a distracting effect of an irrelevant color singleton. Our distraction effect ranged from $21 \mathrm{msec}$ (at display size 5) to $34 \mathrm{msec}$ (at display size 9), very close to the effect sizes reported in Theeuwes (1992).

One potentially troublesome aspect of our design (and that of Theeuwes, 1992) is that the distraction factor was blocked rather than mixed within blocks. Because subjects could predict whether or not an irrelevant color singleton would be present on a given trial, it would have been possible to employ different strategies on different blocks. Thus, what looks like the capture of attention by an irrelevant singleton (the distraction effect) may actually reflect the adoption of a strategy on distraction blocks that happens to result in slower processing in those blocks. To check this possibility, we ran a control experiment (12 paid subjects) that was identical to Experiment 1, except that all blocks contained distractor trials and no-distractor trials, randomly mixed. We found a significant distraction effect in this control experiment $[F(1,11)=10.58$, $p<.01]$, suggesting that the obtained distraction effect was not due to an artifact associated with blocking the distraction factor. ${ }^{4}$

Table 1

Error Rates (in Percent) for Experiment 1

\begin{tabular}{lccc}
\hline & \multicolumn{3}{c}{ Display Size } \\
\cline { 2 - 4 } Condition & 5 & 7 & 9 \\
\hline No distractor & 2.5 & 2.8 & 2.9 \\
Distractor & 2.9 & 2.0 & 3.1 \\
\hline
\end{tabular}

Now that we have replicated the basic effect, we can turn our attention to testing the hypothesis described earlier. That is, even though the target identity (green circle) was known, subjects might have adopted a singleton detection strategy, which, as described earlier, would be susceptible to interference from irrelevant singletons. In other words, instead of searching for the circle, subjects might have searched for the odd shape. The approach of the next two experiments was to discourage such a strategy by including trials in which that strategy would be ineffective.

\section{EXPERIMENT 2}

Under the scenario outlined in the introduction, search for a known target may be accomplished by using either a feature search mode or a singleton detection mode, with the latter but not the former susceptible to interference from singletons on irrelevant dimensions. If the distraction effects reported in Theeuwes (1992) and our Experiment 1 were due to subjects adopting a singleton detection strategy, it should be possible to eliminate the distraction effect by eliminating the effectiveness of this strategy. In the present experiment, we attempted this by including two or three target circles on some trials. Thus we ensured that the target was not reliably a form singleton; in fact, on some trials (three targets with display size 5) there were more target shapes than nontarget shapes. If subjects are indeed able to use a feature search mode that does not rely on detection of differences, the present task should encourage such a strategy.

\section{Method}

Subjects. Sixteen Johns Hopkins University undergraduates (10 male) participated in partial fulfillment of an introductory psychology course requirement. Each subject reported normal or corrected-tonormal visual acuity and normal color vision. Testing took about $50 \mathrm{~min}$.

Stimuli and Procedure. The experiment was very similar to Experiment 1 , except that rather than only one target circle appearing on each trial, up to three targets (each containing a line segment of the same orientation) appeared. This factor, number of targets $(1,2$, or 3$)$, was combined factorially with the other within-block factor, display size $(5,7,9)$. As in Experiment 1 , there were four 144-trial blocks alternating between no-distractor and distractor conditions, preceded by two practice half blocks. The subjects were given the same instructions as before, except that they were told that when more than one target was present, the lines within each target shape would always be the same. Sample displays for twoand three-target trials are shown in Figure 3. One-target trials were identical to those of Experiment 1, depicted in Figure 1.

\section{Results and Discussion}

Once again, RTs greater than $2,000 \mathrm{msec}$ were excluded from analysis, resulting in the removal of considerably less than $1 \%$ of trials. Mean correct RTs were entered into a three-way repeated measures ANOVA with number of targets $(1,2$, or 3 ), distraction (no distractor vs. distractor), and display size $(5,7,9)$ as factors. There were main effects of number of targets $[F(2,30)=34.78, p<.001]$ and of display size $[F(2,30)=28.01, p<.001]$. The 


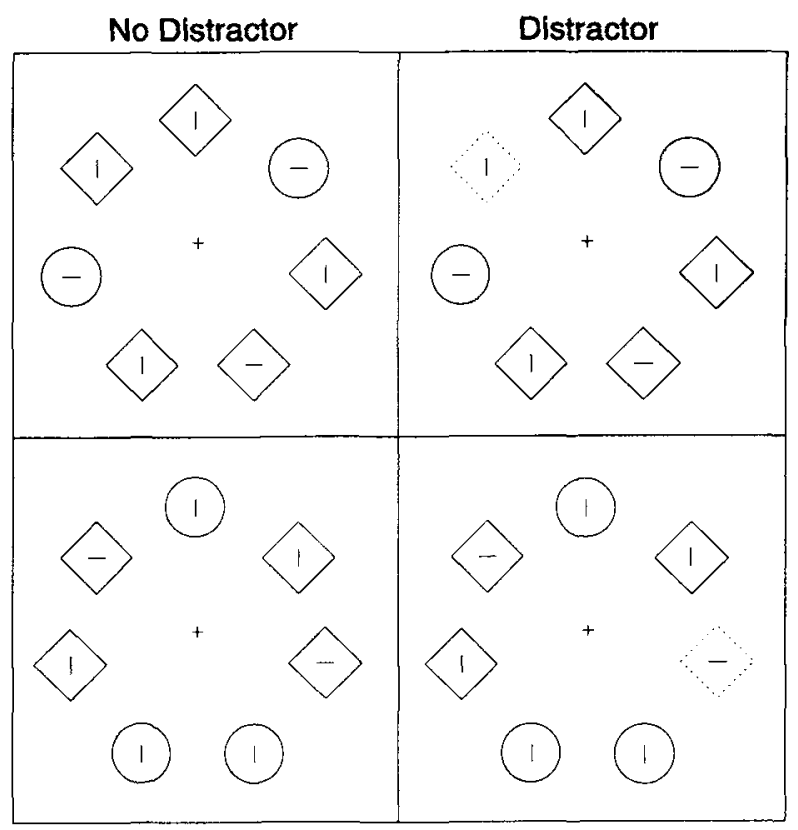

Figure 3. Sample stimulus displays (with display size 7) for Experiment 2. Top panels show two-target trials; bottom panels show three-target trials. One-target trials were the same as in Experiment 1 (see Figure 1).

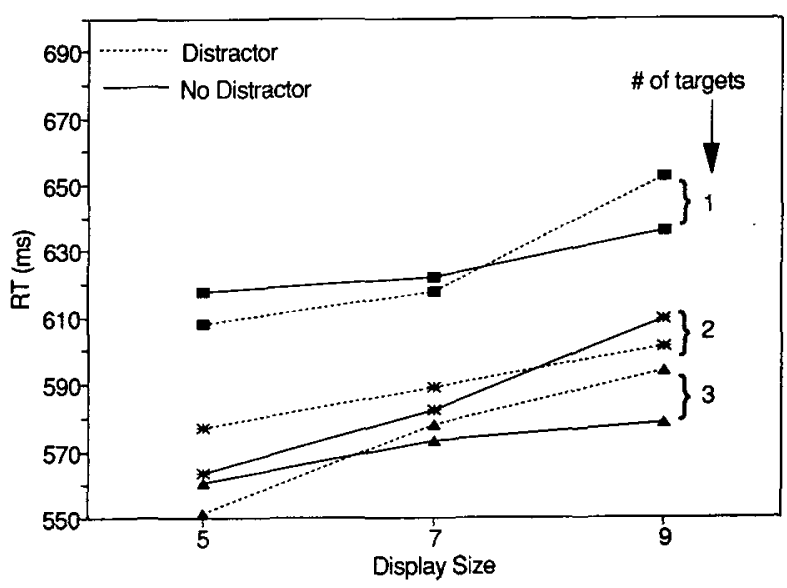

Figure 4. Mean response times, as a function of display size, number of targets, and distractor condition, for Experiment 2.

distraction factor was not significant (overall effect was $3 \mathrm{msec}$ ), nor were any of the interactions. As can be seen in Figure 4, the effect of number of targets is an expected redundancy gain: Performance improves with more targets. This is consistent with both parallel and serial search (Pashler, 1987; Snodgrass \& Townsend, 1980).

The significant display-size effect was explored by computing slopes for each distraction $\times$ number of targets combination; these are shown in Table 2. Although several of the slopes are significantly greater than zero, they are small and in the range that generally has been inter- preted as indicating parallel search (e.g., Treisman \& Gelade, 1980).

Although we are suggesting that search was essentially parallel in Experiment 2, the slopes were significantly greater than zero (as indeed was the slope for the distractor condition in Experiment 1). It is worth considering what problems, if any, this might cause for the interpretation of the (absence of) distraction effects. Theeuwes (1991a) has argued that a distraction effect from an irrelevant singleton will be attenuated in tasks that are performed serially. This argument seems plausible if one assumes that the serial process selects elements randomly, with no effective guidance. Thus in a "relentlessly serial" task (such as a search for a randomly tilted $T$ among randomly tilted Ls; Bergen \& Julesz, 1983; Wolfe et al., 1989), it might not be surprising if one found that an irrelevant color singleton did not produce a distraction effect: Bottom-up (salience) information (as well as feature-based top-down information) would be ineffective for directing attention selectively to the target in such a task, so it is plausible that attention would be directed to elements either at random or solely on the basis of spatial location. Given the significantly greater-than-zero slopes in the present experiment, such an argument might be used to explain why we did not find a distraction effect in this experiment. We find this argument untenable for the following reasons. First, the slopes, although nonzero, are extremely shallow (the steepest slope reported in this paper is $11.5 \mathrm{msec} / \mathrm{element}$, and most of our slopes are well under $10 \mathrm{msec} / \mathrm{element}$ ). Strictly serial searches generally produce much steeper slopes (e.g., 40-60 msec/element in the $T-L$ task of Wolfe et al., $1989 ; 55-80 \mathrm{msec} / \mathrm{element}$ in a rotated $T$ among rotated $I$ s task of Theeuwes, 1990). A strictly serial mechanism would have to move implausibly fast to generate our shallow slopes. Thus, it is clear that something guided attention to the target faster than is possible by a random scan. We know that bottom-up difference signals were not the source of this guidance, because difference signals did not reliably specify the target, and because the highly salient color singleton was apparently ignored. Therefore, we conclude that top-down information about the target features guided attention. That is, subjects were able to employ the feature search mode, with attention being rapidly allocated to the display location containing the known target features (green circle). ${ }^{5}$ Another reason to doubt the argument that serial processing masked a distraction effect in the present experiment comes from Theeuwes's (1992) Experiment 2. In that experiment, Theeuwes found a significant distraction

Table 2

Display-Size Slopes (Milliseconds/Element) for Experiment 2

\begin{tabular}{lccc}
\hline & \multicolumn{3}{c}{ Number of Targets } \\
\cline { 2 - 4 } Condition & 1 & 2 & 3 \\
\hline No distractor & 4.6 & $11.5^{*}$ & 4.4 \\
Distractor & $11.1^{*}$ & $6.1^{*}$ & $10.6^{*}$ \\
\hline
\end{tabular}

Note-Nonasterisked slopes were not significantly different from zero (one-tailed $p>.05$ ). ${ }^{*} p<.01$, one-tailed. 
Table 3

Error Rates (in Percent) for Experiment 2

\begin{tabular}{ccccc}
\hline & \multirow{2}{*}{ No. } & \multicolumn{3}{c}{ Display Size } \\
\cline { 3 - 5 } Condition & Targets & 5 & 7 & 9 \\
\hline No distractor & 1 & 5.5 & 5.2 & 4.2 \\
& 2 & 4.5 & 3.9 & 3.2 \\
\multirow{3}{*}{ Distractor } & 3 & 1.5 & 1.9 & 2.0 \\
& 1 & 5.8 & 4.2 & 5.4 \\
& 2 & 2.4 & 2.1 & 3.8 \\
& 3 & 2.6 & 2.9 & 1.7 \\
\hline
\end{tabular}

effect with a slope of $12.9 \mathrm{msec} / \mathrm{element}$, which is larger than the slopes reported in the present paper. Thus, if the degree of seriality implied by a slope of $12.9 \mathrm{msec} / \mathrm{element}$ was not sufficient to mask the distraction effect in that experiment, it would be difficult to argue that the even less pronounced serial component in the present task masked a distraction effect here.

Subjects' overall error rates ranged from $1.5 \%$ to $6.1 \%$, with a mean of $3.5 \%$. Arcsine-transformed error rates were entered into a three-way repeated measures ANOVA (number of targets $\times$ distraction $\times$ display size). The only significant effect was a main effect of number of targets $[F(2,30)=19.15, p<.001]$. As can be seen in Table 3, subjects made fewer errors with greater number of targets. This result parallels the RT findings and provides evidence against a speed-accuracy tradeoff.

The crucial results of this experiment concern performance in the one-target condition, because the results from this condition are directly comparable to those of Experiment 1 . In the present experiment, trials in the one-target condition were identical to the trials in Experiment 1, except that in the present experiment these trials were embedded in others (with additional targets present) that made a singleton detection strategy ineffective. The overall ANOVA for Experiment 2 (and inspection of Figure 4) indicates that subjects were not distracted by the presence of an irrelevant color singleton. In order to examine the distraction factor specifically in the trials that are comparable to the previous experiment, and to compare the effect of distraction in the two experiments, we conducted an omnibus ANOVA, combining the data from Experiment 1 with data from the one-target condition of Experiment 2 . In this analysis, experiment ( 1 or 2 ) was a between-subjects factor and distraction was a withinsubjects factor. There was a main effect of distraction $[F(1,30)=16.99, p<.001]$. More importantly, there was an interaction between experiment and distraction $[F(1,30)=15.33, p<.001]$, meaning that the effect of distraction was different in the two experiments. An analysis of simple main effects revealed that there was a significant effect of distraction for the Experiment 1 group $[F(1,15)=24.42, p<.001$ (as we know from the original ANOVA on Experiment 1 data)] but no effect of distraction for the Experiment 2 group $[F(1,15)<1]$. The distraction effect was $27 \mathrm{msec}$ for the Experiment 1 group and less than $1 \mathrm{msec}$ for the Experiment 2 group.
The manipulation of number of targets worked precisely as expected; that is, it eliminated the distraction effect, presumably by encouraging subjects to abandon the singleton detection mode. However, the reader might well wonder just why such results were expected. The subjects knew that the multiple targets all contained line segments of the same orientation. Why, then, didn't the subjects simply use a differencing mechanism that would permit all of the multiple targets to pop out-whichever one was selected first would not matter because of the built-in redundancy of the inscribed line segments. To put it differently, it would seem to be a very narrow view of the singleton detection mode if it were to be restricted literally to making one item pop out. In our view, the effectiveness of the multiple-targets manipulation relied upon the relatively small display sizes we used. Assuming that difference signals are based on the mean difference between an element and all other elements, the presence of several targets in a display with relatively few elements will reduce the difference signals of the target(s) substantially. In the extreme case, with three targets in a display of five elements, the targets actually have smaller difference signals than the nontargets. It was our (apparently warranted) assumption that the presence of such trials would encourage subjects to abandon the singleton detection strategy, and employ feature search instead. For larger display sizes, it may be that more than one item at a time can be found by what we have called here the singleton detection mode. If that were to happen, it would be appropriate to refer to this process with a more general term such as discrepancy detection mode.

That no distraction effect was obtained in the present experiment suggests that eliminating the singleton detection strategy allowed for complete top-down selectivity. In other words, when subjects are discouraged from using the singleton detection mode, they apparently can guide attention to a specified target (goal-directed selection) and avoid being distracted by an element that is salient in an irrelevant dimension.

The results of the present experiment provide support for our explanation of Theeuwes's (1992) findings. That is, subjects in that study (and in our Experiment 1) might have adopted a singleton detection strategy, which would be susceptible to distraction by irrelevant singletons, even though the target's identity was known. When we eliminated the effectiveness of such a strategy in Experiment 2, we found no distraction effect, even on trials identical to the trials of Experiment 1. In the next experiment, we sought converging evidence for this explanation.

\section{EXPERIMENT 3}

In the previous experiment, we attempted to eliminate the effectiveness of the singleton detection strategy by adding additional target shapes to some displays. In the present experiment, we took a different approach toward the same goal. We sought to eliminate the singleton detection strategy by adding, on some trials, additional 
unique forms. That is, instead of all nontargets being the same shape (diamonds), on some trials one or two of the nontargets were themselves unique with respect to form (a square and/or a triangle). As in Experiment 1, the target was always a unique form (the only circle in the field), but in the present experiment there were other unique forms as well. Because the target shape was not the only item unique with respect to form, the target would no longer have reliably the highest difference signal, and the singleton detection mode would frequently deliver the wrong elements. If, as is suggested by the results of Experiment 2 , subjects are able to use a feature search mode, directing attention to a specific shape, the present task should encourage such a strategy. This should result in a search that is not affected by the presence of a color singleton.

\section{Method}

Subjects. Sixteen Johns Hopkins University undergraduate and graduate students (10 male) participated and were paid $\$ 5$. Each subject reported normal or corrected-to-normal visual acuity and normal color vision. Testing took about $50 \mathrm{~min}$.

Stimuli and Procedure. The only difference between this experiment and Experiment 1 concerns the shapes of the nontargets. In the earlier experiment, all nontargets were diamonds. In the present experiment, zero, one, or two of the nontargets were changed to a unique shape. We refer to this factor as the number of unique forms. When the number of unique forms was one, only the target circle was a form singleton (that is, no nontargets were changed from the standard diamond shape). Thus, trials in this condition were identical to trials in Experiment 1, and to the single-target condition of Experiment 2; typical trials are shown in Figure 1. The other two types of trial in the present experiment are shown

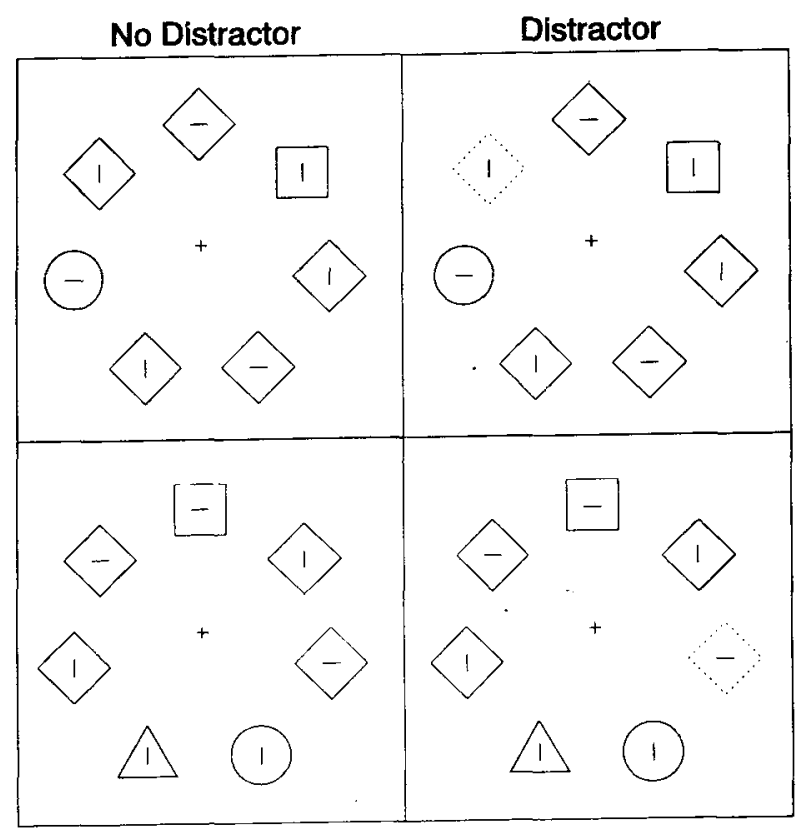

Figure 5. Sample stimulus displays (with display size 7) for Experiment 3. Top panels show two-unique-forms trials; bottom panels show three-unique-forms trials. One-unique-form trials were the same as in Experiment 1 (see Figure 1).

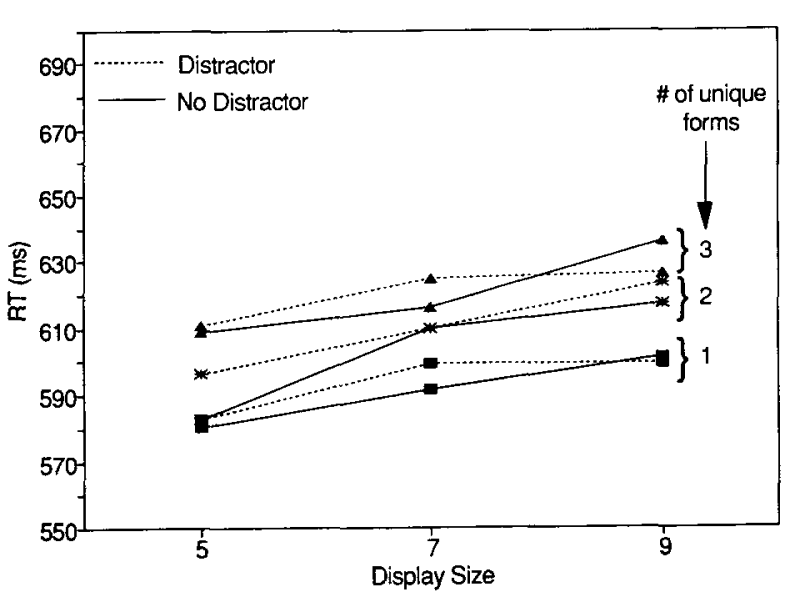

Figure 6. Mean response times, as a function of display size, number of unique forms, and distractor condition, for Experiment 3.

in Figure 5. The top panels depict displays in the two-unique-forms condition. In this condition, one of the nontarget shapes was randomly chosen to be either a square or a triangle, instead of the standard diamond shape of the nontargets. Thus, both the target shape and one of the nontarget shapes were unique forms. The bottom panel shows sample displays when the number of unique forms was three. In these trials, one nontarget was a square and another nontarget was a triangle. The squares had the same size as did the diamonds. Triangles were upward-pointing and equilateral, $1.6^{\circ}$ on a side. Nontarget squares and triangles were always green. The factor of number of unique forms $(1,2$, or 3$)$ was combined factorially with the other within-block factor, display size $(5,7,9)$. As in the previous experiments, there were four 144-trial blocks alternating between no-distractor and distractor conditions, preceded by two practice half blocks. The subjects were given the same instructions as in Experiment 1, except that they were also informed of the additional possible nontarget shapes. As in the previous experiments, line segments inscribed in the nontargets were randomly selected to be either horizontal or vertical. Orientations were selected independently for each nontarget element, including both the standard nontargets (diamonds) and the unique-form nontargets (squares and circles).

\section{Results and Discussion}

As in the previous experiments, RTs greater than $2,000 \mathrm{msec}$ were excluded from analysis, resulting in the removal of considerably less than $1 \%$ of trials. Mean correct RTs were entered into a three-way repeated measures ANOVA with number of unique forms $(1,2$, or 3$)$, distraction (no distractor vs. distractor), and display size (5, $7,9)$ as factors. There were main effects of number of unique forms $[F(2,30)=10.77, p<.001]$ and of display size $[F(2,30)=9.39, p<.001]$. Most importantly, the 3-msec distraction effect was not significant $[F(1,15)$ $<1]$. None of the interactions attained significance. As can be seen in Figure 6, the effect of number of unique forms reflects the fact that subjects were slower on trials with heterogeneous nontarget shapes. This is a commonly observed effect (e.g., Duncan \& Humphreys, 1989).

Display-size slopes were computed for each subject; the mean slopes for each condition are presented in Table 4 . The slopes were significantly greater than zero in all but 
Table 4

Display-Size Slopes (Milliseconds/Element) for Experiment 3

\begin{tabular}{lccc} 
& \multicolumn{3}{c}{ Number of Unique Forms } \\
\cline { 2 - 4 } Condition & 1 & 2 & 3 \\
\hline No distractor & $5.2^{*}$ & $8.5^{*}$ & $6.7^{*}$ \\
Distractor & $4.1^{*}$ & $6.8 \dagger$ & 3.8 \\
\hline
\end{tabular}

${ }^{*} p<.05$, one-tailed. $\dagger p<.01$, one-tailed.

Table 5

Error Rates (in Percent) for Experiment 3

\begin{tabular}{ccccc}
\hline & $\begin{array}{c}\text { No. } \\
\text { Unique } \\
\text { Condition }\end{array}$ & \multicolumn{3}{c}{ Display Size } \\
\cline { 4 - 5 } & Forms & 5 & 7 & 9 \\
\hline No distractor & 1 & 3.7 & 2.4 & 2.6 \\
& 2 & 3.1 & 2.6 & 3.1 \\
Distractor & 3 & 1.8 & 3.4 & 2.8 \\
& 1 & 3.8 & 2.8 & 1.3 \\
& 2 & 2.4 & 2.4 & 2.6 \\
& 3 & 2.4 & 2.4 & 4.2 \\
\hline
\end{tabular}

one condition. However, these slopes were all small (well under $10 \mathrm{msec} / \mathrm{element}$ ) and in the range generally assumed to reflect parallel processing. (See the discussion following Experiment 2.)

The subjects' overall error rates ranged from $0.5 \%$ to $5.7 \%$, with a mean of $2.8 \%$. Mean error rates by condition are shown in Table 5. An ANOVA on the subjects' arcsinetransformed error rates revealed no significant effects.

As in the previous experiment, the finding of principal interest is the absence of any distractor effect-that is, of any effect of an irrelevant color singleton. To ensure that this holds for the one-form-singleton condition (which is the condition that is directly comparable to Experiment 1), and to compare the effects of distraction across these two experiments, we performed an omnibus ANOVA with experiment ( 1 or 3 ) as a between-subjects factor and distraction as a within-subjects factor. There was a main effect of distraction $[F(1,30)=12.75, p<.01]$ and an interaction between experiment and distraction $[F(1,30)=$ $8.80, p<.01]$, meaning that the effect of distraction differed between the two experiments. An analysis of simple main effects revealed that the $27-\mathrm{msec}$ distraction effect of the Experiment 1 group was significant (as we know from previous ANOVAs) but that the 3-msec distraction effect for the Experiment 3 group was not significant $[F(1,15)<1]$.

These results add converging evidence to the findings from the previous experiment. Under certain circumstances, subjects can perform a parallel search for a known shape target and not be distracted by the presence of a singleton on an irrelevant dimension.

\section{GENERAL DISCUSSION}

We began with a puzzle. Why is there sometimes a distracting effect of irrelevant-dimension singletons during visual search even when the target and distractor identi- ties are known in advance? (Such an effect was found by Theeuwes, 1992; see also Pashler, 1988, for a small effect that was not tested for significance.) Our proposed solution was that even when target identity is known subjects may sometimes search not for a specific shape, but for the odd shape. This strategy, which we called the singleton detection mode, is assumed to be susceptible to interference from singletons on irrelevant dimensions.

In Experiment 1, we replicated Theeuwes's (1992) findings. During search for a circle target among diamond nontargets, subjects were distracted by the presence of a nontarget that differed in color from the other elements. The logic of the two subsequent experiments was to make the singleton detection mode ineffective, thereby forcing subjects to search for the specific target feature. In Experiment 2 , we accomplished this by adding redundant targets on some trials, so that the target was not reliably a form singleton. In Experiment 3, we changed one or two nontargets to create irrelevant unique forms on some trials. Both manipulations were apparently successful. There were no distracting effects of color singletons in these experiments, demonstrating that top-down selectivity is indeed possible during parallel search.

Our conclusion is that goal-directed selection of a specific known featural identity may override stimulus-driven capture caused by salient featural singletons. Theeuwes's (1992) findings that irrelevant singletons cause distraction decrements cannot be used to argue that selection is based solely on stimulus-driven factors. When the structure of the task discourages the singleton detection mode (as in the present Experiments 2 and 3), subjects apparently have no difficulty in directing attention to the target feature (feature search) and thereby avoiding distraction from color singletons.

The findings reported here are consistent with the view that subjects in our Experiment 1 and in Theeuwes's (1992) experiments employed the singleton detection mode, even though the feature search mode was available. Why would subjects do this? If top-down selectivity for a specific feature value was possible, and this would have eliminated the distracting effect of the irrelevant color singleton, why didn't subjects make use of it, employing the feature search mode? At this point, we can only acknowledge that this is an interesting question. An obvious possibility is that subjects employed the singleton detection mode because it was easier and because they could. It is conceivable that the feature search mode is more cognitively demanding, requiring, for example, effortful concentration on the target feature. In our Experiment 1, the less demanding singleton detection mode may have served adequately well, delivering at most one nontarget (the irrelevant color singleton) before delivering the target. The singleton detection mode was made less efficient by the manipulations in Experiments 2 and 3. For example, in the worst case of Experiment 3, the singleton detection mode might deliver three nontargets (the irrelevant color singleton, and the two nontargets with unique forms) before finding the target. Apparently this degree of inefficiency was sufficient to induce subjects to employ the 
feature search mode. It may be possible to induce subjects to employ the feature search mode in other ways as well, using instructional or motivational manipulations.

There are both methodological and theoretical implications in the present study. The methodological point is that subjects may not always do what experimenters tell them to do. Even when a target identity is known and subjects can perform feature search, they may adopt alternative strategies if such strategies are available. In order to ensure that subjects do not instead perform singleton detection, the experimenter must eliminate the usefulness of such a strategy.

From a theoretical standpoint, the results presented here are also of interest. Most current theories of attentional selection posit a role for the top-down selection of visual attributes. The theory of Bundesen (1990), for example, claims that a visual element's attentional weight is a function of the "pertinence" of the element's perceptual category. Pertinence is defined in relation to task demands (e.g., target identity in a search). Perceptual categories include location, color, and shape; thus, the theory makes no qualitative distinction between selection by location and selection by other attributes. Similarly, the theory of Duncan (1980) allows for selection by location as well as by other attributes.

Rapid conjunction search (e.g., Egeth, Virzi, \& Garbart, 1984) seems to demand explanation in terms of topdown attentional selection, and theories designed to account for such findings do posit top-down selection mechanisms. Recent versions of feature integration theory (Treisman, 1988) allow for elements on a master map to receive inhibition from feature maps known to correspond to nontarget elements. The guided search model (Cave \& Wolfe, 1990; Wolfe et al., 1989) allows for activation of elements possessing target features. These theories would be in difficulty if top-down selection were possible only for spatial location, and not for other visual attributes. The present findings that top-down selection can override stimulusdriven attentional capture are therefore important.

The present findings are also relevant to the contingent involuntary orienting hypothesis of Folk et al. (1992). According to this hypothesis, stimulus-driven capture of attention depends on attentional control settings, which are a function of task demands. Although our experiments were not designed specifically to test this hypothesis, the results are, in a broadly thematic way, consistent with it. As described in the introduction, the vulnerability of the singleton detection mode to distraction by irrelevant singletons could be considered to reflect the adoption of an attentional set for a discrepant item; this set would also adequately describe the irrelevant color singleton, which would therefore be an appropriate attentional target. Our manipulations in Experiments 2 and 3 could be considered to be forcing subjects to adopt a narrower attentional set, one that does not include the irrelevant color singleton. In this framework, the distraction effects observed by Theeuwes (1991a, 1992) and in the present Experiment 1 should not be considered to be entirely stimulus driven.
Rather, such effects are consistent with the attentional state of the observer.

The present experiments may be useful in interpreting Folk et al.'s (1992) examination of the level at which the attentional settings can be configured. In their Experiment 4, Folk et al. (1992) examined whether interference from the precue depended only on being of the same type (static discontinuity) as the target, or whether interference depended on the specific feature value, such as, for example, the color red. They found that a different color (green) precue also inhibited processing of a subsequent red target, and thus they argued that the attentional control settings operate at the level of the type (static vs. dynamic) of discontinuity that is relevant for finding the target.

From the present perspective, we would suggest that narrower attentional control settings (i.e., for a specific feature value rather than for a class of discontinuity) may in fact be possible, but that they did not emerge in Folk et al.'s (1992) task. Because the target was a red character and all nontargets were always white, subjects could employ either the feature search mode (searching for red) or the singleton detection mode (searching for the odd color). If subjects did employ the singleton detection mode, we would expect an irrelevant singleton (of any color) in the precue to also capture attention. On the basis of our results, we would predict that if subjects were forced into the feature search mode, say by presenting the red target among variously colored nontargets (analogous to our Experiment 3), the green precue would no longer be disruptive. This prediction remains to be tested.

A final theoretical issue raised by the present experiments concerns the nature of the singleton detection mode. It was suggested earlier that when in this mode subjects search for an element that differs from its neighbors, but that the dimension on which the element differs is unavailable prior to selection. What type of representation could subserve such a search strategy? In the model proposed by Sagi and Julesz (1984), the visual scene is preattentively represented as a map of differences. That is, elements that differ from their neighbors on certain conspicuous features are represented, but their feature identities are not represented preattentively. Such a map would be an obvious choice as a representation subserving the singleton detection mode. However, because this map codes differences but not identities, it could not subserve the feature search mode, which was apparently employed by our subjects in Experiments 2 and 3.

The "activation map" of guided search theory (Cave $\&$ Wolfe, 1990) fares better. This representation receives both bottom-up and top-down activation from feature maps. The bottom-up component reflects the salience of each element; the top-down component represents additional activation from feature maps corresponding to known features of the target. Under this model, the feature search mode and the singleton detection mode would both involve a search through elements with large values on the activation map. The feature search mode would correspond to situations in which there were large top- 
down activations during search for a known target. The singleton detection mode would correspond to situations in which little or no top-down activation was involved. This would be the case during search for an unknown target (i.e., the target could be a circle among diamonds or a diamond among circles, as in Theeuwes, 1991a). However, it could also be the case during search for a known target (as in Theeuwes, 1992, and in the present Experiment 1), if subjects failed to supply top-down activation.

Yantis and Hillstrom (in press) propose a model in which an "attentional priority map" receives input from both stimulus-driven and goal-directed processes. As with guided search, under this model both feature search and singleton detection would be based on the same representation; the difference between the modes would then reflect only the relative contributions of the stimulusdriven and goal-directed processes to activation on the priority map.

In conclusion, we have shown that irrelevant featural singletons may or may not cause distraction during parallel search for a known target, depending on the search strategy employed. Feature-specific top-down information is indeed available to guide attention rapidly to potential targets, thus avoiding distraction from irrelevant singletons, but this feature-specific information is apparently not used when salience alone can adequately solve the task. Our experiments provide a warning that human observers have capabilities that may be masked by strategies made possible by rather subtle aspects of experimental design.

\section{REFERENCES}

Bergen, J. R., \& Julesz, B. (1983). Parallel versus serial processing in rapid pattern discrimination. Nature, 303, 696-698.

Bravo, M. J., \& Nakayama, K. (1992). The role of attention in different visual-search tasks. Perception \& Psychophysics, 51, 465-472.

Broadbent, D. (1958). Perception and communication. Oxford: Pergamon.

Bundesen, C. (1990). A theory of visual attention. Psychological Review, 97, 523-547.

CAve, K. R., \& Wolfe, J. M. (1990). Modelling the role of parallel processing in visual search. Cognitive Psychology, 22, 225-271.

DunCaN, J. (1980). The locus of interference in the perception of simultaneous stimuli. Psychological Review, 87, 272-300.

Duncan, J. (1985). Visual search and visual attention. In M. I. Posner \& O. S. M. Marin (Eds.), Attention and performance XI (pp. 85106). Hillsdale, NJ: Erlbaum.

DunCAN, J., \& HUMPHREYs, G. W. (1989). Visual search and stimulus similarity. Psychological Review, 96, 433-458.

Egeth, H. E., VIRZI, R. A., \& Garbart, H. (1984). Searching for conjunctively-defined targets. Journal of Experimental Psychology: Human Perception \& Performance, 10, 32-39.

Folk, C. L., REMington, R. W., \& Johnston, J. C. (1992). Involuntary covert orienting is contingent on attentional control settings. Journal of Experimental Psychology: Human Perception \& Performance, 18, 1030-1044.

Folk, C. L., Remington, R. W., \& Johnston, J. C. (1993). Contingent attentional capture: A reply to Yantis (1993). Journal of Experimental Psychology: Human Perception \& Performance, 19, 682-685.

IvEs, H. E. (1912). Studies of the photometry of lights of different colours: I. Spectral luminosity curves obtained by the equality of brightness photometer and the flicker photometer under similar conditions. Philosophical Magazine, 24, 149-188.
JuLEsz, B. (1981). Textons, the elements of texture perception, and their interactions. Nature, 290, 91-97.

Koch, C., \& Ullman, S. (1985). Shifts in selective visual attention: Towards the underlying neural circuitry. Human Neurobiology, 4, 219-227.

LABERGE, D., \& BRown, V. (1989). Theory of attentional operations in shape identification. Psychological Review, 96, 101-124.

Nothdurft, H.-C. (1992). Feature analysis and the role of similarity in preattentive vision. Perception \& Psychophysics, 52, 355-375.

Pashler, H. (1987). Detecting conjunctions of color and form: Reassessing the serial search hypothesis. Perception \& Psychophysics, 41, 191-201.

Pashler, H. (1988). Cross-dimensional interaction and texture segregation. Perception \& Psychophysics, 43, 307-318.

Posner, M. I., Snyder, C. R. R., \& Davidson, B. J. (1980). Attention and the detection of signals. Journal of Experimental Psychology: General, 109, 160-174.

SAGI, D., \& JULESZ, B. (1984). Detection versus discrimination of visual orientation. Perception, 13, 619-628.

SNOdGrass, J. G., \& Townsend, J. T. (1980). Comparing parallel and serial models: Theory and implementation. Journal of Experimental Psychology: Human Perception \& Performance, 6, 330-354.

TheEuwes, J. (1990). Perceptual selectivity is task dependent: Evidence from selective search. Acta Psychologica, 74, 81-99.

Theeuwes, J. (1991a). Cross-dimensional perceptual selectivity. Perception \& Psychophysics, 50, 184-193.

TheEuwes, J. (1991b). Exogenous and endogenous control of attention: The effect of visual onsets and offsets. Perception \& Psychophysics, 49, 83-90.

Theeuwes, J. (1992). Perceptual selectivity for color and form. Perception \& Psychophysics, 51, 599-606.

Treisman, A. (1988). Features and objects: The Fourteenth Bartlett Memorial Lecture. Quarterly Journal of Experimental Psychology, 40A, 201-237.

Treisman, A., \& Gelade, G. (1980). A feature integration theory of attention. Cognitive Psychology, 12, 97-136.

Treisman, A., \& Souther, J. (1985). Search asymmetry: A diagnostic for preattentive processing of separable features. Journal of Experimental Psychology: General, 114, 285-309.

WINER, B. J. (1971). Statistical principles in experimental design (2nd ed.). New York: McGraw-Hill.

Wolfe, J. M., CAVE, K. R., \& Franzel, S. L. (1989). Guided search: An alternative to the feature integration model for visual search. Journal of Experimental Psychology: Human Perception \& Performance, $15,419-433$.

YaNTIS, S. (1993a). Stimulus-driven attentional capture. Current Directions in Psychological Science, 2, 156-161.

YANTIS, S. (1993b). Stimulus-driven attentional capture and attentional control settings. Journal of Experimental Psychology: Human Perception \& Performance, 19, 676-681.

Yantis, S., \& HLlstrom, A. (in press). Stimulus-driven attentional capture: Evidence from equiluminant visual objects. Journal of $E x-$ perimental Psychology: Human Perception \& Performance.

YANTIS, S., \& Jonides, J. (1984). Abrupt visual onsets and selective attention: Evidence from visual search. Journal of Experimental Psychology: Human Perception \& Performance, 10, 601-621.

YANTIS, S., \& JoNIDES, J. (1990). Abrupt visual onsets and selective attention: Voluntary versus automatic allocation. Journal of Experimental Psychology: Human Perception \& Performance, 16, 121-134.

\section{NOTES}

1. Folk et al. (1992) question whether control of attention is ever purely stimulus driven. They argue that the ability of salient stimuli (including abrupt onsets) to capture attention depends on the attentional control settings induced by task demands. This issue remains controversial (see Folk et al., 1993, Yantis, 1993b).

2. The observed difference in accuracy between the distractor and no-distractor conditions when the target was specified was $2.2 \%$. This simple effect was not tested for significance, but the significant interaction between the distraction factor and the target specification factor 
indicates that this effect was smaller than the $9.4 \%$ effect when the target was unspecified. This was consistent with Pashler's (1988) hypothesis that irrelevant singletons should interfere substantially only when the target feature is unspecified.

3. Theeuwes (1992) also found performance decrements due to an irrelevant form singleton when a yellowish-green circle target was among yellowish-red circle distractors (the "color condition"). Obtaining an effect in this condition depended on finding suitably desaturated colors such that the form difference was more salient than the color difference. We restricted our attention to the form condition.

4. In the control experiment, the distraction effect was small $(3 \mathrm{msec})$ at display size 5, but otherwise comparable to the effect found in the first experiment ( 18 and $25 \mathrm{msec}$ for display sizes 7 and 9, respectively). This is reflected in the significant distraction $\times$ display-size effect
$[F(2,22)=6.2, p<.01]$. Although this interaction was unexpected, the basic results of the control experiment were the same as those of Experiment 1, and provide evidence that the distraction effect is robust.

5 . The fact that slopes are nonzero suggests that the guidance is not perfect. In the guided search model (Cave \& Wolfe, 1990; Wolfe et al., 1989), small slopes in essentially parallel searches are explained by the presence of noise in the activation map. Even when preattentive information guides attention to the target very efficiently, occasionally a nontarget location will be selected first. With increasing numbers of elements in the display, it becomes more likely that a nontarget activation will exceed threshold by chance.

(Manuscript received March 8, 1993; revision accepted for publication August 7, 1993.) 